CERN-TH/96-183

FTUAM 96/28

hep-ph/9607405

\title{
Orbifold-induced $\mu$ term and electroweak symmetry breaking
}

\author{
A. Brignole ${ }^{1}$, L.E. Ibáñez ${ }^{2}$ and C. Muñoz ${ }^{2}$ \\ ${ }^{1}$ Theory Division, CERN \\ CH-1211 Geneva 23, Switzerland \\ ${ }^{2}$ Departamento de Física Teórica C-XI, \\ Universidad Autónoma de Madrid, \\ Cantoblanco, 28049 Madrid, Spain
}

\begin{abstract}
It is known that a Higgs $\mu$ term can be naturally generated through the Kähler potential in orbifold string models in which one of the three compactified complex planes has order two. In this class of models explicit expressions for both the $\mu$ parameter and the soft SUSY-breaking parameters can be obtained under the assumption that the goldstino is an arbitrary linear combination of the fermionic partners of the dilaton $S$ and all the moduli $T_{i}, U_{i}$. We apply this picture to the MSSM and explore the consistency of the obtained boundary conditions with radiative gauge symmetry breaking. We find that consistency with the measured value of the top-quark mass can only be achieved if the goldstino has a negligible dilatino component and relevant components along the $T_{3}, U_{3}$ moduli associated to the order-two complex plane.
\end{abstract}


1. The Minimal Supersymmetric extension of the Standard Model (MSSM) contains two kinds of mass terms: a set of soft supersymmetry-breaking terms, including scalar and gaugino mass terms, and a globally supersymmetric Higgs mass term, the so-called $\mu$ term. If SUSY breaking originates from a super-Higgs mechanism in an underlying supergravity theory, the gravitino gets a mass $m_{3 / 2}$ and soft parameters $\mathcal{O}\left(m_{3 / 2}\right)$ are usually generated. In addition, if the supergravity Kähler potential contains appropriate terms bilinear in the Higgs fields, an effective $\mu$ parameter $\mathcal{O}\left(m_{3 / 2}\right)$ can be generated as well [1]. Although not unique, this way to generate a $\mu$ term is particularly attractive because $\mu$ becomes directly related to SUSY breaking and thus stands essentially on the same footing as the other mass parameters.

In the restricted and motivated class of effective supergravity theories corresponding to 4-D superstring compactifications, specific patterns of soft terms emerge under the assumption that SUSY breaking is due to non-vanishing $F$-components for the dilaton $(S)$ and moduli $\left(T_{i}, U_{i}\right)$ fields [2]-[0]. Such an assumption implies that the goldstino is a linear combination of the fermionic partners of the dilaton and the moduli, the coefficients of the combination just measuring the relative contribution of each field to SUSY breaking. We recall that, in the case of orbifold models, the set of Kähler moduli $T_{i}$ always includes the three diagonal Kähler moduli $T_{1}, T_{2}, T_{3}$ associated to the three compactified complex planes, whereas the number of complex structure moduli $U_{i}$ can be at most three. Here we will follow the approach of [由, [], where the soft parameters are expressed in terms of the gravitino mass $m_{3 / 2}$, the angles specifying the (free) goldstino direction and the modular weights of the matter fields. However, we recall that some ambiguity affects the results for the $\mu$ parameter and the associated soft $B$ parameter, depending on the source of the $\mu$ term itself. In this respect, an interesting and predictive class of models consists of orbifold models in which one of the three compactified complex planes (the third, say) has order two. We will focus on such a class of models and denote by $T_{3}$ and $U_{3}$ the Kähler and complex structure moduli associated to that plane. It was found in [8, 9] that the Kähler potential corresponding to $T_{3}$ and $U_{3}$, and to charged untwisted fields $C_{1}, C_{2}$ in conjugate representations associated to the same plane, has the form

$$
K=-\log \left[\left(T_{3}+T_{3}^{*}\right)\left(U_{3}+U_{3}^{*}\right)-\left(C_{1}+C_{2}^{*}\right)\left(C_{1}^{*}+C_{2}\right)\right] .
$$

After SUSY breaking, a certain effective $\mu$ term [9] is induced for the fields $C_{1}$ and $C_{2}$, which we will identify here with the electroweak Higgs fields. Under the assumption that this is the main source of the $\mu$ term and that SUSY breaking is dilaton/moduli-dominated, simple expressions for $\mu$ and $B$ in terms of goldstino angles can be derived [7]. Therefore a quite predictive scenario is obtained, with explicit and correlated expressions for the full set of mass parameters. The aim of the present letter is to apply such a scenario to the MSSM and check its consistency with radiative electroweak symmetry breaking and the constraints on the top quark mass.

2. In the following we will assume that the MSSM can be obtained from a string model of the kind mentioned above and regard the resulting tree-level expressions for the soft and $\mu$ parameters as boundary conditions given at some high scale $M_{X}$. We will then use one-loop RGEs to evolve the parameters down to the electroweak scale $m_{Z}$, where we will impose the standard requirement of electroweak symmetry breaking and evaluate the top mass. Such 'leading-order' procedure, which neglects e.g. high- and low-energy threshold corrections, will be sufficient for our purpose. 
In the numerical evaluation, we will identify $M_{X}$ with the apparent unification scale of gauge couplings $M_{G} \sim 3 \times 10^{16} \mathrm{GeV}$, and check that the main conclusions do not change even if $M_{X}$ is allowed to take larger values.

We recall that the MSSM can be described in terms of a superpotential $W=$ $\mu H_{1} H_{2}+h_{t} Q_{3} U_{3}^{c} H_{2}$ and a soft Lagrangian of the form

$$
\mathcal{L}_{\text {soft }}=\left(\frac{1}{2} M_{a} \lambda^{a} \lambda^{a}-\mu B H_{1} H_{2}-h_{t} A_{t} Q_{3} U_{3}^{c} H_{2}+\text { h.c. }\right)-\sum_{\alpha} m_{\alpha}^{2}\left|\phi_{\alpha}\right|^{2},
$$

where we have neglected Yukawa couplings different from the top one $h_{t}$. The scalar potential, restricted to the real parts $h_{1}, h_{2}$ of the neutral Higgs fields, has the standard form:

$$
V\left(h_{1}, h_{2}\right)=\left(m_{H_{1}}^{2}+\mu^{2}\right) h_{1}^{2}+\left(m_{H_{2}}^{2}+\mu^{2}\right) h_{2}^{2}+\left(\mu B h_{1} h_{2}+\text { h.c. }\right)+\frac{1}{8}\left(g^{2}+g^{\prime 2}\right)\left(h_{1}^{2}-h_{2}^{2}\right)^{2},
$$

where $m_{H_{1}}^{2}$ and $m_{H_{2}}^{2}$ are the Higgs soft masses. Under our assumptions the MSSM mass parameters are functions of the goldstino direction, which can be parametrized[ by an angle $\theta$ and additional parameters $\Theta_{i}$ [0]. For instance, the goldstino components along the fermionic partners of the dilaton $S$ and the moduli $T_{1}, T_{2}, T_{3}$, $U_{3}$ are proportional to $\sin \theta, \cos \theta \Theta_{1}, \cos \theta \Theta_{2}, \cos \theta \Theta_{3}, \cos \theta \Theta_{3}^{\prime}$, respectively. We also recall that the parameters $\Theta_{i}$ are constrained by $\Theta_{1}^{2}+\Theta_{2}^{2}+\ldots+\Theta_{3}^{2}+\Theta_{3}^{\prime 2}=1$, where ... correspond to possible contributions from additional $T$-type or $U$-type moduli. However, it is important to realize that the only goldstino parameters directly relevant for electroweak symmetry breaking are $\theta, \Theta_{3}$ and $\Theta_{3}^{\prime}$. First of all, such parameters control the boundary conditions for the Higgs mass parameters. This is not surprising, of course, since the Higgs fields are associated to the third complex plane. Using the specific form (1) for the third-plane Kähler potential, one finds that such mass parameters at the high scale $M_{X}$ can be expressed as [7]

$$
\begin{aligned}
& m_{H_{1}}^{2}=m_{H_{2}}^{2}=m_{3 / 2}^{2}\left(1-3 \cos ^{2} \theta\left(\Theta_{3}^{2}+\Theta_{3}^{\prime 2}\right)\right) \\
& \mu=m_{3 / 2}\left(1+\sqrt{3} \cos \theta\left(\Theta_{3}+\Theta_{3}^{\prime}\right)\right) \\
& B=m_{3 / 2} 2 \frac{\left(1+\sqrt{3} \cos \theta \Theta_{3}\right)\left(1+\sqrt{3} \cos \theta \Theta_{3}^{\prime}\right)}{\left(1+\sqrt{3} \cos \theta\left(\Theta_{3}+\Theta_{3}^{\prime}\right)\right)} .
\end{aligned}
$$

Second, the renormalization of the above parameters down to $m_{Z}$ does not involve further goldstino angles. Indeed, renormalization effects are controlled by $M_{a}, A_{t}$ and the specific combination $m_{\Sigma}^{2} \equiv m_{Q_{3}}^{2}+m_{U_{3}}^{2}+m_{H_{2}}^{2}$ of Higgs and stop soft masses. The corresponding boundary conditions at $M_{X}$ are [7]

$$
M_{a}=-A_{t}=m_{3 / 2} \sqrt{3} \sin \theta, m_{\Sigma}^{2}=m_{3 / 2}^{2} 3 \sin ^{2} \theta .
$$

More comments on renormalization effects will be given below. Here we recall for completeness that the above results for $A_{t}$ and $m_{\Sigma}^{2}$ ('sum rule') include the reasonable assumption that the coupling $h_{t} Q_{3} U_{3}^{c} H_{2}$ corresponds to a renormalizable coupling also in the underlying string theory. Specifically, this implies that $Q_{3}$ and $U_{3}$ correspond to untwisted fields or twisted fields with overall modular weight -1 associated to the first and second complex planes. Notice, however, that we only

\footnotetext{
${ }^{1}$ We neglect complex phases and set the cosmological constant to zero.
} 
need the combination $m_{\Sigma}^{2}$ and not the individual masses $m_{Q_{3}}^{2}$ and $m_{U_{3}}^{2}$, which depend on the goldstino parameters $\Theta_{1}, \Theta_{2}, \ldots$, as well as on partial modular weights. We will not discuss the remaining squark and slepton masses either, since such a discussion would introduce further model dependence, i.e. a generic dependence on $\Theta_{1}, \Theta_{2}, \ldots$, and on individual modular weights [7]. Such model-dependent features do not interfere with the issue of electroweak breaking, which is the main subject of the present analysis.

Before renormalizing the mass parameters, we summarize the situation at the high scale $M_{X}$. We recall that the tree-level Higgs potential (3) at $M_{X}$ has a flat direction along $h_{1}=-h_{2}$, independently of the goldstino direction [4]. This property follows from the equality of the three coefficients

$$
m_{H_{1}}^{2}+\mu^{2}=m_{H_{2}}^{2}+\mu^{2}=\mu B
$$

which control the quadratic part of the Higgs potential. At this stage the only constraint on the space of goldstino angles comes from the requirement that the Higgs potential be bounded along the orthogonal direction $h_{1}=h_{2}$, i.e. that the common value of the three parameters in (6) be non-negative;:

$$
\left(1+\sqrt{3} \cos \theta \Theta_{3}\right)\left(1+\sqrt{3} \cos \theta \Theta_{3}^{\prime}\right) \geq 0 .
$$

Therefore, at the classical level, for any goldstino direction satisfying the stability condition (7) one finds a continuous set of $S U(2) \times U(1)$ breaking vacua characterized by $\tan \beta=\left\langle h_{2}\right\rangle /\left\langle h_{1}\right\rangle=-1$ and sliding Higgs vev's. In order to remove such vacuum degeneracy we will adopt the conventional point of view that the electroweak vacuum is determined radiatively, i.e. by logarithmic and $h_{t}$-dependent quantum corrections which renormalize the parameters in the Higgs potential from $M_{X}$ down to $m_{Z}$. In so doing we will encounter very different situations depending on the goldstino direction.

The RG evolution of the parameters down to $m_{Z}$ can be performed numerically in a straightforward way. Analytical formulae can be derived as well, e.g. along the lines of [11]. Rather than giving the solutions in detail, here we only note that the dependence of the renormalized parameters on the goldstino angles can be easily inferred from the corresponding boundary conditions and the coupled system of RGEs. In particular, it is easy to see that $M_{a}$ and $A_{t}$ remain proportional to $\sin \theta$ and $m_{\Sigma}^{2}$ to $\sin ^{2} \theta$ at any scale. Therefore, since these parameters drive the RG evolution of $m_{H_{1}}^{2}, m_{H_{2}}^{2}$ and $B$, the additive renormalization of the latter parameters is controlled by $\sin \theta$ only?. On the other hand, the $\mu$ parameter has a simple multiplicative renormalization as usual. In conclusion, the Higgs potential mass

\footnotetext{
${ }^{2}$ An analogous result holds of course in other models, e.g. GUTs, having a conjugate pair of Higgs fields that could be identified with $C_{1}$ and $C_{2}$ in (11).

${ }^{3}$ Notice that such a constraint does not prevent $m_{H_{1}}^{2}$ and $m_{H_{2}}^{2}$ from being negative for some goldstino directions. For example, they are indeed negative in the third-plane-dominated SUSYbreaking scenarios, which will turn out to be phenomenologically favoured.

${ }^{4}$ A similar situation was found for specific goldstino directions and in a slightly different context in $[10]$.

${ }^{5}$ We are neglecting terms proportional to $g^{\prime 2} \sum_{\alpha} Y_{\alpha} m_{\alpha}^{2}$ in the RGEs for scalar masses, which may induce a further small and model-dependent renormalization of $m_{H_{1}}^{2}$ and $m_{H_{2}}^{2}$, and can be considered as part of our uncertainties. Notice however that such terms are absent when squark and slepton masses have universal boundary conditions, e.g. in the dilaton-dominated scenario and possibly others. Even in the general case, moreover, both $m_{\Sigma}^{2}$ and $m_{H_{1}}^{2}+m_{H_{2}}^{2}$ are unaffected.
} 
parameters at the scale $m_{Z}$ have the form

$$
\begin{aligned}
m_{H_{1}}^{2} & =m_{3 / 2}^{2}\left(1-3 \cos ^{2} \theta\left(\Theta_{3}^{2}+\Theta_{3}^{\prime 2}\right)+\sin ^{2} \theta c_{1}\right) \\
m_{H_{2}}^{2} & =m_{3 / 2}^{2}\left(1-3 \cos ^{2} \theta\left(\Theta_{3}^{2}+\Theta_{3}^{\prime 2}\right)+\sin ^{2} \theta c_{2}\right) \\
\mu & =m_{3 / 2}\left(1+\sqrt{3} \cos \theta\left(\Theta_{3}+\Theta_{3}^{\prime}\right)\right) c_{\mu} \\
B & =m_{3 / 2}\left[2 \frac{\left(1+\sqrt{3} \cos \theta \Theta_{3}\right)\left(1+\sqrt{3} \cos \theta \Theta_{3}^{\prime}\right)}{\left(1+\sqrt{3} \cos \theta\left(\Theta_{3}+\Theta_{3}^{\prime}\right)\right)}+\sin \theta c_{B}\right]
\end{aligned}
$$

where $c_{i}$ are calculable renormalization coefficients 0 which depend on $\log \left(M_{X} / m_{Z}\right)$ and (apart from $c_{1}$ ) on the boundary value for $h_{t}$, which we denote by $h_{t}^{0}$. The $c_{i}$ 's do not depend on the goldstino angles, which are explicitly factored out in (8).

For a given goldstino direction satisfying (7), radiative electroweak symmetry breaking occurs if the Higgs potential mass parameters at $m_{Z}$ satisfy the basic conditions

$$
\begin{aligned}
& \left(m_{H_{1}}^{2}+\mu^{2}\right)\left(m_{H_{2}}^{2}+\mu^{2}\right)<(\mu B)^{2} \\
& \left(m_{H_{1}}^{2}+\mu^{2}\right)+\left(m_{H_{2}}^{2}+\mu^{2}\right) \geq 2|\mu B|
\end{aligned}
$$

which express the instability of the Higgs potential at the origin and its boundedness along the D-flat directions, respectively. Since $m_{3 / 2}$ is only an overall factor in (9), the relevant parameter to be adjusted is just $h_{t}^{0}$. Typically, the conditions (9) are simultaneously satisfied only for a narrow range of $h_{t}^{0}$ values, which is mapped onto an even narrower range of $h_{t}\left(m_{Z}\right)$ values. For $h_{t}^{0}$ in the favourable range the Higgs potential develops an $S U(2) \times U(1)$ breaking minimum. The corresponding $\tan \beta$ can be computed froml

$$
\sin 2 \beta=\frac{-2 \mu B}{m_{H_{1}}^{2}+m_{H_{2}}^{2}+2 \mu^{2}},
$$

where $m_{3 / 2}$ drops out again. Finally one can evaluate the corresponding top mass parameter $\hat{m}_{t}=v h_{t}\left(m_{Z}\right)|\sin \beta|$, with $v \simeq 174 \mathrm{GeV}$. In conclusion, the symmetry breaking requirement allows us to associate a certain range of $\hat{m}_{t}$ values to any given goldstino direction. Rather than translating $\hat{m}_{t}$ values into pole mass values, we will be content with a qualitative comparison with the experimental constraints and consider $\hat{m}_{t}$ values smaller than $150 \mathrm{GeV}$ as phenomenologically unacceptable. We will see that this simple and conservative criterion will be sufficient to put strong constraints on the different SUSY-breaking scenarios.

As an additional comment, we note that we have exploited only one of the two relations that follow from the minimization of the Higgs potential, i.e. eq. (10) above. The second relation can be written as

$$
m_{Z}^{2}=\frac{m_{H_{2}}^{2}-m_{H_{1}}^{2}}{\cos 2 \beta}-\left(m_{H_{1}}^{2}+m_{H_{2}}^{2}+2 \mu^{2}\right)
$$

${ }^{6}$ Alternatively to a numerical evaluation, one could use e.g. the analytical formulae in the second ref. of 11] and express the $c_{i}$ 's as $c_{1}=3 g, c_{2}=h+3(e+f-k)-1, c_{\mu}=q, c_{B}=\sqrt{3}(r+s) / q$, where the opposite sign convention for gaugino masses has been taken into account. Note that our sign conventions in (2) lead to RGEs for $A$ and $B$ having signs as in $d A / d t \sim-A-M$, $d B / d t \sim-A-M$, where $t=\log M_{X}^{2} / Q^{2}$.

${ }^{7}$ As a side remark, we note that in the present approach the signs of all input parameters are given and therefore the output parameter $\tan \beta$ will have a $\operatorname{sign}$ too. The correct $\operatorname{sign}$ can be obtained using e.g. $\tan \beta=(1-\cos 2 \beta) / \sin 2 \beta$, where $\cos 2 \beta=-\sqrt{1-\sin ^{2} 2 \beta}$ as a consequence of $m_{H_{2}}^{2}<m_{H_{1}}^{2}$. 
with $\beta$ given in (10). Since $m_{3 / 2}^{2}$ is an overall factor in the right-hand side, the above equation formally establishes a one-to-one correspondence between $m_{Z}^{2} / m_{3 / 2}^{2}$ and $h_{t}^{0}$ for any given goldstino direction. Therefore, it seems that the minimization conditions in principle allow the scale $m_{3 / 2}$ to be fixed as a function of $h_{t}^{0}$ (or $\hat{m}_{t}$ ). However, such correspondence is of little practical use, mainly because a narrow range of allowed $h_{t}^{0}$ values corresponds to a wide (formally infinite) range of $m_{3 / 2}$ values and the mapping itself is subject to several uncertainties. Therefore we will not rely explicitly on such $m_{3 / 2}-\hat{m}_{t}$ correlation.

3. For convenience, we will describe our results in terms of three categories of goldstino directions, each one being a special case of the next: i) pure dilaton SUSY breaking; ii) dilaton/overall-modulus SUSY breaking; iii) dilaton/moduli SUSY breaking.

i) Pure dilaton SUSY breaking. This scenario corresponds to the limit $\cos \theta \rightarrow$ 0 , i.e. to the two inequivalent cases $\sin \theta= \pm 1$. The stability condition (7) is automatically satisfied. The renormalized mass parameters (8) read

$$
\begin{aligned}
m_{H_{1}}^{2} & =m_{3 / 2}^{2}\left(1+c_{1}\right) \\
m_{H_{2}}^{2} & =m_{3 / 2}^{2}\left(1+c_{2}\right) \\
\mu & =m_{3 / 2} c_{\mu} \\
B & =m_{3 / 2}\left(2 \pm c_{B}\right) .
\end{aligned}
$$

The numerical analysis shows that for $\sin \theta=+1$ the symmetry-breaking requirements (9) select the initial top Yukawa in the range $h_{t}^{0} \sim(0.11-0.13)$. Correspondingly, one finds $h_{t}\left(m_{Z}\right) \sim(0.4-0.5), \tan \beta \sim-(1-3)$ and too small a top mass $\hat{m}_{t}<70 \mathrm{GeV}$. For $\sin \theta=-1$, the situation is even worse. Proper symmetry breaking occurs for $h_{t}^{0} \sim(0.04-0.05)$, corresponding to $h_{t}\left(m_{Z}\right) \sim 0.2, \tan \beta \sim-(1-1.2)$ and $\hat{m}_{t}<25 \mathrm{GeV}$. Therefore the specific $\mu$ mechanism considered here is incompatible with the dilaton SUSY-breaking scenario. This result is consistent e.g. with the findings of ref. [12], where the dilaton scenario phenomenology was studied using the boundary condition $B=2 m_{3 / 2}$ whereas $\mu$ was treated as a free parameter. In such an approach the value of $\mu$ required by radiative symmetry breaking and leading to an acceptable top mass was much larger than the specific 'orbifold' value $\mu=m_{3 / 2}$ used here.

ii) Dilaton/overall-modulus SUSY breaking. This scenario corresponds to generic $\theta$ and to $\Theta_{1}=\Theta_{2}=\Theta_{3}=1 / \sqrt{3}$ (implying in particular $\Theta_{3}^{\prime}=0$ ), i.e. to the limit in which only the dilaton and a symmetric combination of the Kähler moduli $T_{1}, T_{2}$, $T_{3}$ participate to SUSY breaking. Again, the stability condition (7) is automatically satisfied. The renormalized mass parameters (8) now read

$$
\begin{aligned}
m_{H_{1}}^{2} & =m_{3 / 2}^{2} \sin ^{2} \theta\left(1+c_{1}\right) \\
m_{H_{2}}^{2} & =m_{3 / 2}^{2} \sin ^{2} \theta\left(1+c_{2}\right) \\
\mu & =m_{3 / 2}(1+\cos \theta) c_{\mu} \\
B & =m_{3 / 2}\left(2+\sin \theta c_{B}\right)
\end{aligned}
$$

and the symmetry-breaking requirements (9) associate a certain range of $h_{t}^{0}$ values to each $\theta$. Such $h_{t}^{0}$ values are quite small in most of the $\theta$ range, typically implying a top mass below $100 \mathrm{GeV}$. For $-30^{\circ} \lesssim \theta \lesssim 30^{\circ}$ one can get somewhat higher values for 
$h_{t}^{0}(\sim 0.3-0.6)$, leading to $h_{t}\left(m_{Z}\right)$ values close to 1 . In such cases, however, $|\tan \beta|$ turns out to be very close to 1 and one always finds $\hat{m}_{t} \lesssim 140 \mathrm{GeV}$, the maximum value being reached for $\theta \sim-20^{\circ}$. Therefore also this class of goldstino directions seems incompatible with radiative breaking and top mass value. Notice, however, that the situation improves when one moves from dilaton- to moduli- dominated SUSY breaking.

iii) Dilaton/moduli SUSY breaking. This is the most general scenario, corresponding to generic values for $\theta$ and $\Theta_{i}$, i.e. a generic goldstino direction. Actually we expect the potentially interesting directions to have small $\sin \theta$, since the analysis of scenario (ii) has shown that an improvement can be obtained only when the goldstino has a suppressed dilatino component. To obtain a further improvement one should depart from the overall-modulus direction and explore different ways to distribute the non-dilatonic SUSY-breaking contribution among the individual moduli. In this respect, we could expect $T_{3}$ and $U_{3}$ to give a peculiar contribution to SUSY breaking, since the third complex plane plays a special role in the class of models considered here. For instance, one can think of the two limiting and complementary situations in which those moduli dominate SUSY breaking or do not participate at all. These will be special and important examples of the general scenario considered here. Before reporting the general results, we note that the goldtsino directions are now subject to two preliminary constraints. One is the stability condition (7), which is no longer automatic. An additional constraint arises from the sum rule relating the boundary values of stop and Higgs soft masses. If one requires $m_{Q_{3}}^{2}+m_{U_{3}}^{2} \geq 0$ at $M_{X}$ in order to avoid instabilities of the scalar potential along charged and coloured directions, the formulae for $m_{\Sigma}^{2}=m_{Q_{3}}^{2}+m_{U_{3}}^{2}+m_{H_{2}}^{2}$ and $m_{H_{2}}^{2}$ in (5) and (4) lead to the constraint

$$
\Theta_{3}^{2}+\Theta_{3}^{\prime 2} \geq 1-\frac{2}{3 \cos ^{2} \theta} .
$$

This inequality was automatically satisfied for the special goldstino directions considered in (i) and (ii). Here it constrains the parameters $\Theta_{3}, \Theta_{3}^{\prime}$ when $\cos ^{2} \theta>2 / 3$, or equivalently the angle $\theta$ when $\Theta_{3}^{2}+\Theta_{3}^{\prime 2}<1 / 3$. We recall that $\Theta_{3}^{2}+\Theta_{3}^{\prime 2} \leq 1$ by definition.

The general form of the renormalized Higgs potential mass parameters has already been written above (8). Once the radiative symmetry-breaking conditions are imposed, we find that the top mass $\hat{m}_{t}$ is too small in the case of negligible $\Theta_{3}, \Theta_{3}^{\prime}$, i.e. when the third plane moduli do not participate significantly to SUSY breaking. This result is partly due to the above constraint (14), which prevents us from reaching the small $\sin \theta$ region $\$$. One can get $\hat{m}_{t} \gtrsim 150 \mathrm{GeV}$ only if $\sin \theta$ is sufficiently small and at the same time both $\Theta_{3}$ and $\Theta_{3}^{\prime}$ are non-negligible. Roughly speaking, the former condition allows us to reach sufficiently large values for $h_{t}^{0}$ (anyway smaller than $\sim 0.6$, but this is enough), whereas the latter one allows us to displace $|\tan \beta|$ from 1 at the same time. For a heuristic explanation of the latter point, we recall that $|\tan \beta| \gg 1$ corresponds to $|\sin 2 \beta| \ll 1$, which typically requires $|B| \ll m_{3 / 2}$. For small $\sin \theta$ this cannot happen in the overall-modulus case $\left(\Theta_{3}=1 / \sqrt{3}, \Theta_{3}^{\prime}=0\right)$ because $B$ is dominated by the boundary condition $B=2 m_{3 / 2}$, whereas it can happen for simultaneously non-vanishing $\Theta_{3}, \Theta_{3}^{\prime}$. As an example of favourable goldstino direction, we can quote e.g. the limiting case $\Theta_{3}=\Theta_{3}^{\prime}=1 / \sqrt{2}$, where $T_{3}$ and $U_{3}$ are the only moduli contributing to SUSY

${ }^{8}$ If that constraint is relaxed, some points with large $\hat{m}_{t}$ values can be found, but we will not take this possibility into account in the present discussion. 
breakingf. Large values of $\hat{m}_{t} \sim 150-175 \mathrm{GeV}$ can be reached for $\theta \sim\left(170^{\circ}-180^{\circ}\right)$, the maximum $\hat{m}_{t}$ corresponding to $\theta \sim 176^{\circ}$. A similar situation can be found in a nearby region and in another not equivalent region where $\operatorname{sign}\left(\Theta_{3} \Theta_{3}^{\prime}\right)<0$. For instance, the latter region does not include the values $\Theta_{3}=-\Theta_{3}^{\prime}=1 / \sqrt{2}$ because the constraint (7) is violated for small $\sin \theta$. Also, there the largest $\hat{m}_{t}$ values are obtained for $\cos \theta>0$ instead of $\cos \theta<0$. However we refrain from giving a detailed correspondence between goldstino angles $\left\{\theta, \Theta_{3}, \Theta_{3}^{\prime}\right\}$ and values of $\hat{m}_{t}$, since such mapping is affected by several uncertainties"एण. We rather stress again the main conclusion of the exploration in the general case, that is the possibility to reach phenomenologically acceptable values of the top mass in a region of goldstino angles characterized by small $\sin \theta$ and simultaneously non-negligible $\Theta_{3}, \Theta_{3}^{\prime}$.

We conclude this section by mentioning some qualitative features of the SUSY spectra corresponding to the favourable region of goldstino angles. In such a region the boundary conditions (5) for gaugino masses and $A_{t}$ are smaller than $m_{3 / 2}$, due to the smallness of $\sin \theta$, whereas the boundary conditions for stop masses and $\mu$ are generically $\mathcal{O}\left(m_{3 / 2}\right)$. This hierarchy tends to be preserved at low energy. In particular, the lightest chargino and neutralinos are mainly gaugino-like and typically lighter than the stop squarks, whereas the heaviest ones are mainly higgsino-like and have masses of the same order as the stop squarks. The gluino and the heavy Higgses tend to stay in an intermediate range. We stress again that the precise mass ratios will depend on the specific goldstino direction. We also recall that general predictions for the remaining squark and slepton masses cannot be made unless additional model-dependent information is provided, as mentioned in section 2 .

4. In summary, we have applied the general results of the dilaton/moduli SUSYbreaking scenario to the MSSM, using at the same time a specific and predictive mechanism for generating $\mu$ available in a class of orbifold models. Such models have an order-two compactified complex plane and induce an effective $\mu$ parameter through Higgs bilinear terms in the Kähler potential. Starting from such 'stringy' boundary conditions for the MSSM mass parameters, we have studied whether the combined requirement of radiative electroweak symmetry breaking and a sufficiently large top mass constrain the goldstino direction, i.e. the relative size of the dilaton and moduli $F$-terms. We have found that the above phenomenological requirements cannot be satisfied either in the dilaton dominated scenario or in the mixed dilaton/overall-modulus scenario. The only SUSY-breaking scenario compatible with such constraints requires a suppressed dilaton contribution and important (often dominant) contributions from the moduli fields associated to the same order-two plane as the Higgs fields. On the phenomenological side such a restricted SUSYbreaking scenario implies certain features for the SUSY spectra. On the theoretical side the above conclusion can perhaps be taken as an indication guiding the search for explicit models of SUSY breaking.

\footnotetext{
${ }^{9}$ It is interesting to apply the radiative breaking criterion to the explicit simple model built in the first ref. of [10], which includes a Kähler potential of the form (11). There the only modulus contributing to SUSY breaking is the diagonal combination of $T_{3}$ and $U_{3}$, corresponding just to the favourable values $\Theta_{3}=\Theta_{3}^{\prime}=1 / \sqrt{2}$. However in such a model the dilaton SUSY-breaking contribution is non-negligible. It corresponds to $\sin \theta=-1 / \sqrt{3}(\cos \theta=-\sqrt{2 / 3})$, which leads to too small a top mass, $\hat{m}_{t}<80 \mathrm{GeV}$.

${ }^{10}$ We recall that, among other effects, we have neglected string loop corrections. These may become relevant precisely in the small $\sin \theta$ region, but they are very model-dependent.
} 


\section{References}

[1] G.F. Giudice and A. Masiero, Phys. Lett. B206 (1988) 480.

[2] L.E. Ibáñez and D. Lüst, Nucl. Phys. B382 (1992) 305.

[3] V.S. Kaplunovsky and J. Louis, Phys. Lett. B306 (1993) 269.

[4] A. Brignole, L.E. Ibáñez and C. Muñoz, Nucl. Phys. B422 (1994) 125 [Erratum: B436 (1995) 747].

[5] S. Ferrara, C. Kounnas and F. Zwirner, Nucl. Phys. B429 (1994) 589 [Erratum: B433 (1995) 255].

[6] T. Kobayashi, D. Suematsu, K. Yamada and Y. Yamagishi, Phys. Lett. B348 (1995) 402;

P. Brax and M. Chemtob, Phys. Rev. D51 (1995) 6550;

E. Dudas, S. Pokorski and C.A. Savoy, Phys. Lett. B369 (1996) 255.

[7] A. Brignole, L.E. Ibáñez, C. Muñoz and C. Scheich, hep-ph/9508258, to appear in Z. Phys. C.

[8] G. Lopes-Cardoso, D. Lüst and T. Mohaupt, Nucl. Phys. B432 (1994) 68.

[9] I. Antoniadis, E. Gava, K.S. Narain and T.R. Taylor, Nucl. Phys. B432 (1994) 187.

[10] A. Brignole and F. Zwirner, Phys. Lett. B342 (1995) 117;

A. Brignole, F. Feruglio and F. Zwirner, Phys. Lett. B356 (1995) 500.

[11] L.E. Ibáñez and C. Lopez, Nucl. Phys. B233 (1984) 511;

L.E. Ibáñez, C. Lopez and C. Muñoz, Nucl. Phys. B256 (1985) 218.

[12] R. Barbieri, J. Louis and M. Moretti, Phys. Lett. B312 (1993) 451. 